\title{
QM/QM Study of the Coverage Effects on the Adsorption of Amino-Cyclopentene at the Si(100) Surface
}

\author{
HUGO R. R. SANTOS, ${ }^{1}$ GREgORI UJAQUE, ${ }^{2}$ MARIA J. RAMOS, ${ }^{1}{ }^{\text {JOSÉ }}$ A. N. F. GOMES \\ ${ }^{I}$ REQUIMTE, Departamento de Química, Faculdade de Ciências, Universidade do Porto, \\ Rua do Campo Alegre, 687, 4169-007 Porto, Portugal \\ ${ }^{2}$ Unitat de Química Física, Departament de Química, Edifici Cn., \\ Universitat Autònoma de Barcelona, 08193 Bellaterra, Catalonia, Spain
}

Received 12 December 2005; Revised 20 February 2006; Accepted 21 February 2006

DOI 10.1002/jcc.20513

Published online 18 September 2006 in Wiley InterScience (www.interscience.wiley.com).

\begin{abstract}
In this work, we have tested 30 different adsorption situations in several coverage scenarios for the 1amino-3-cyclopentene (ACP) molecule on the $\mathrm{Si}(100)$ surface. We have used a five-spot testing zone inserted in the high-level part of a quantum-mechanical/quantum-mechanical study performed in a big cluster. By defining several different scenarios, each one with a typical adsorption energy, we were able to understand in detail the process of surface functionalization. We are able to justify why the functionalization of this silicon surface achieves only a coverage of $\sim 0.5 \mathrm{ML}$ (half monolayer) and why the completely covered surface should be thermodynamically impossible to obtain.
\end{abstract}

(C) 2006 Wiley Periodicals, Inc. J Comput Chem 27: 1892-1897, 2006

Key words: $\mathrm{Si}(100)$; ACP; adsorption; coverage

\section{Introduction}

Theoretical adsorption studies are often performed and adsorption energies are calculated with no account for the coverage effects that may happen at the surface. Single molecule adsorption on a clean surface does not include the fact that real adsorption processes must be affected by the presence of other already adsorbed molecules. The interactions between the latter can lead to the formation of defects and disorganization and prevent complete coverage of the surface. Obviously, it would be better to be able to create perfect monolayers with no defects. ${ }^{1-3}$ Therefore, it is of the utmost importance to study and define what is happening at the molecular level at the surface, when we are adsorbing many molecules with a view to form monolayers.

Trying to understand the processes involved in the monolayer formation with computational studies is difficult to accomplish mainly because of limitations in the size of cluster that we can build while still getting accurate energies for the adsorption processes. Large systems can be studied with molecular mechanics, ${ }^{4,5}$ but adsorption energies obtained from those calculations are not appropriate because of the oversimplification of the system and disregard of the explicit electronic parts. Medium-sized systems can be represented with semiempirical calculations ${ }^{6-9}$ but even in this case the associated energy errors are too large for the study of most of the molecular adsorption processes. Only relatively heavy DFT $^{10-15}$ or higher level calculations ${ }^{16,17}$ can give results accurate enough for most purposes. However, in these cases the systems' sizes are usually too small to correctly represent a surface area big enough to perform different adsorption situations on already occupied surfaces. Periodic system calculations $^{18}$ could be of some help here but since they are basically $a b$ initio calculations, the periodic cells cannot once again be very large because of CPU time restrictions. Additionally, if we are studying a single defect on a surface that same defect will be repeated at a cell's length from it in all directions with possible consequences in the results. In this work, we try to circumvent these problems by using the hybrid quantum-mechanical/quantum-mechanical (QM/QM) ONIOM methodology. ${ }^{19-21}$ In this way, we can concentrate the computationally heavier calculations in the most important parts of the system, i.e. where the adsorption occurs, while still representing a sufficiently large surface area with a lower level and less computationally intensive method. We have previously used this methodology to simply represent the clean silicon(100) surface, as a first test and

Correspondence to: J. A. N. F. Gomes; e-mail: jfgomes@reit.up.pt Contract/grant sponsor: HPC-Europa; contract/grant number: RII3-CT2003-506079

Contract/grant sponsor: European Community-Research Infrastructure Action of the FP6

Contract/grant sponsor: Spanish MEC; contract/grant number: CTQ200509000-C02-01 
methodology efficiency verification, ${ }^{22}$ and also in single molecule adsorption calculations for an experimental situation test. ${ }^{23}$ In both of these situations, we found that the combination of the high-level DFT method using the hybrid B3LYP functional ${ }^{24}$ with the Shape and Hamiltonian Consistent (SHC*) basis $\operatorname{set}^{25,26}$ and the low-level semiempirical Austin Method (AM1) ${ }^{27}$ gave very good results, comparable to experimental values and theoretical calculations using computationally heavier methods. Moreover, by manipulating the number of atoms in the higher and lower-level layers, we can adjust this method to be useful in much larger systems and still require only relatively short calculation times.

In a previous work, experimentalists attached ssDNA to the $\mathrm{Si}(100)$ surface by functionalizing the surface with the 1-amino3-cyclopentene (ACP) molecule and using sulfo-succinimidyl 4( $N$-maleimidomethyl) cyclohexane-1-carboxylate to link the free amino group of the adsorbed ACP to the $5^{\prime}$-thiol-modified DNA strands. ${ }^{28}$ From an experimental point of view, the perfectly functionalized surface would be the one with ACP molecules attached to all the $\mathrm{Si}(100)$ surface dimers through the $\mathrm{C}=\mathrm{C}$ double bonds ( $\mathrm{C}$-adsorbed) and with the amino groups pointing out from the surface in a trans conformation, i.e. almost perfectly perpendicular to the surface. ${ }^{28}$ The perfectly covered surface, with no defects, would allow to get a better reproducibility of the results and a better protection against small molecules like oxygen $^{29}$ and water ${ }^{30}$ that are known to oxidize the $\mathrm{Si}(100)$ surface. $^{31}$

In this work, we study the coverage effects that influence the adsorption of $\mathrm{ACP}$ on the $\mathrm{Si}(100)$ surface. In a previous study, we have confirmed that the ACP molecule can bind to the surface through the carbon-carbon double bond (C-adsorbed, as shown in detail in Fig. 1), through the amino group (Nadsorbed) or even through both groups at the same time $(\mathrm{CN}-$ adsorbed). ${ }^{23}$ Even though it is impossible to study all the orientations and combinations of ACP molecules on the surface, we have studied an adequate number of possibilities, which allowed us to draw conclusions relating to the system's behaviour; namely, we can now have an idea of the probability to obtain a perfect surface having defined that we are only interested in the trans-ACP conformation, C-adsorbed and perfectly organized at the surface.

There are some scanning tunneling microscopy (STM) studies of cyclopentene adsorbed on the $\mathrm{Si}(100)$ surface that show that the typical distance between adsorbed cyclopentene molecules is roughly double the one found between silicon dimers. $^{32,33}$ This means that cyclopentene adsorbs roughly on a $0.5 \mathrm{ML}$ (half monolayer) surface coverage. It has also been shown previously that the $\mathrm{C}$-adsorbed forms of ACP are very similar to the adsorbed cyclopentene. ${ }^{23}$ This may be already an indication that the ACP case may not be very different.

\section{Computational Details}

In this work, we tried to address the situation by simulating a surface area big enough to contain 21 silicon dimers disposed in three rows with seven dimers each. We used a hybrid quantummechanical/quantum mechanical cluster method (ONIOM) ${ }^{19-21}$ to perform our calculations on the six layers thick with a total of 273 silicon atoms cluster. The central five dimers were the testing positions, treated in the high-level methodology with DFT, basically those spots in which we tried the combinations of adsorbed and nonadsorbed ACP molecules. The peripheral dimers are always occupied by $\mathrm{C}$-adsorbed-trans-ACP molecules, and we used this knowledge to represent the effect of the completely occupied surface with the exception of the small testing site. This outer part was treated with the lower-level method, using the semiempirical AM1. This is what Figure 2 shows, with the large spheres representing the central high-level part and the grey sticks representing the outer low-level part. As we have already demonstrated in a previous work, ${ }^{23}$ the bottom surface of the cluster is also dimerized to compensate for the tension forces caused by the dimerization at the silicon upper surface. This allows for a complete cluster relaxation without any constraints and resulting in a completely flat surface. There are also 104 hydrogen atoms compensating the broken valence at the bottom and sides of the cluster.

Since the ACP density on the full covered surface is very high, the cluster would relax in a somewhat bent, unrealistic geometry. Consequently, we were forced to fix the fifth silicon layer (second counting from the bottom) and some of the silicon atoms in two of the lateral faces of the cluster. By doing this, we avoided the bending and still allowed for some internal cluster relaxation that did occur when the dimers on the surface changed their geometries from tilted to symmetrical upon ACP adsorption. All the atoms in the high-level layer were free to move.

Throughout the article, we have represented the central row by a sequence of "A" (short for ACP) and "_" when there is an ACP molecule adsorbed or a clean spot, respectively. Moreover, while we use an uppercase letter for a high-level ACP, we use a lowercase "a" for the low-level ones. So a full surface is represented by aAAAAAa as shown in Figure 2, or e.g. we can have a aA_A_Aa structure like both the structures shown in Figure 3. Furthermore, for simplicity we also numbered all the positions of the central row of the cluster from 1 to 7 counting from the left-hand side, when looked at from the specific point of view shown in Figure 3. Therefore, the five central testing spots included in the high-level layer are numbered from 2 to 6 .

All calculations were performed with the Gaussian03 and Gaussview software package. ${ }^{34}$

\section{Results and Discussion}

Contrary to previous work where we only found one C-adsorbed ACP conformation, ${ }^{23}$ here we have obtained two different conformations that are relatively similar in terms of energy. Figure 3 shows a clear example of this situation where we can observe that the central molecule can have the amino group closer (top) or farther (bottom) from the surface. These conformations differ by $2 \mathrm{kcal} \mathrm{mol}^{-1}$ and were spotted in several situations throughout our calculations, depending on the starting point geometry. The bottom structure with the amino group farther from the surface was consistently the most stable as well as the one that we had found previously. ${ }^{23}$ However, in another article using a different methodology, 


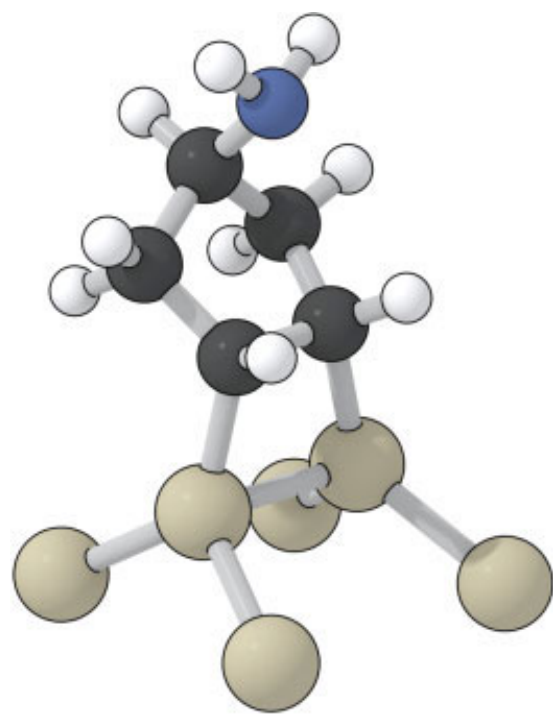

Figure 1. Trans-ACP molecule C-adsorbed to a $\mathrm{Si}(100)$ surface dimer.

though it is not very clear from the images, it seems that the top structure was found as the most stable. ${ }^{35}$ For consistency of the results, we decided to use only the bottom, most stable, conformation in all the calculations.

Table 1 shows the results obtained for the adsorption of one ACP molecule on the empty silicon surface followed by the data that concerns the adsorption of the ACP on a three-spot hole on the $\mathrm{Si}$ surface. What we can conclude from this information is that the three central positions of the testing zone are basically equivalent and that the adsorption energy in this situation is of around $-39 \mathrm{kcal} \mathrm{mol}^{-1}$. In our previous work, with a smaller cluster and without any other ACP molecules adsorbed in the lateral rows, we have achieved a $-40 \mathrm{kcal} \mathrm{mol}^{-1}$ for the adsorption energy of a single ACP molecule also using the SHC* basis

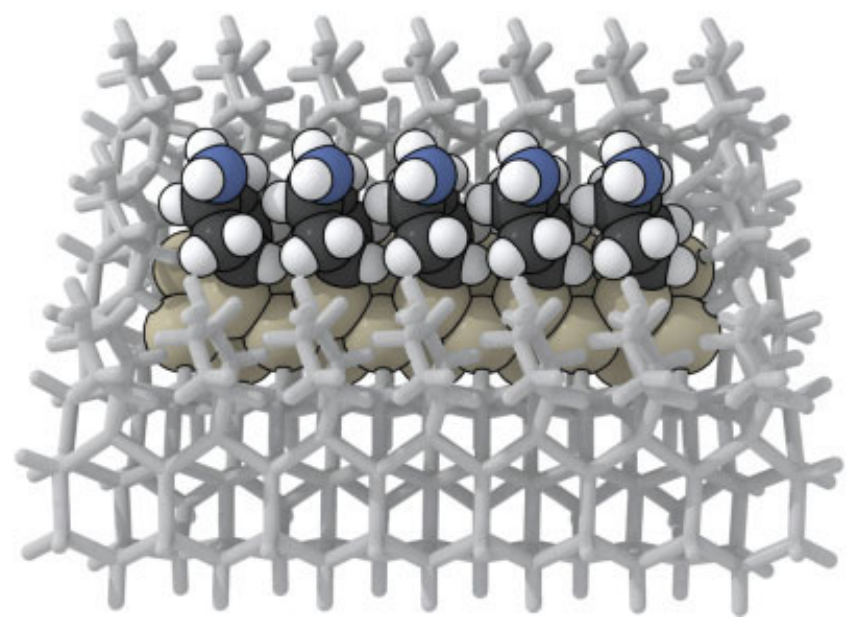

Figure 2. Fully covered cluster (AAAAA). Large spheres represent the high-level part, while grey sticks represent the low-level part. set. $^{23}$ The difference between these results is within the error inherent to the calculations, and this means that there is not a measurable interference between ACP molecules adsorbed right next to each other in parallel rows. More importantly, we can observe from the last three results that even if we have only one hole left on each side of the adsorbed molecule the adsorption energy is basically the same as for the completely clean surface. This points to the fact that adsorption of ACP molecules on a $\mathrm{Si}(100)$ surface can reach a $0.5 \mathrm{ML}$ coverage with no observed interference between adsorbed molecules. Obviously, the dynamics of the process might be affected but we are not dealing with that in this specific work. What we know is that adsorbing a molecule in a completely clean surface or in a near half coverage one should have, ideally, the same adsorption energies. This is also in agreement with previous theoretical works where it was found that the $0.5 \mathrm{ML}$ coverage results were very similar to the adsorption of a single ACP molecule on a clean surface. ${ }^{35}$

Table 2 shows the results obtained for the adsorption of one $\mathrm{ACP}$ molecule between previously adsorbed molecules and one or two empty spaces. The first thing that we can observe is that the first two results differ from all the other similar combinations by $-9 \mathrm{kcal} \mathrm{mol}^{-1}$. To understand this, we must recall that our cluster has seven dimers per row and so when we are adsorbing in the sixth position we only have another low-level ACP molecule at the seventh and last position. This low-level ACP molecule is not fixed and is free to move and this allows it to get farther from the central part and give some extra space for the adsorbing molecule. We can also see that if we are

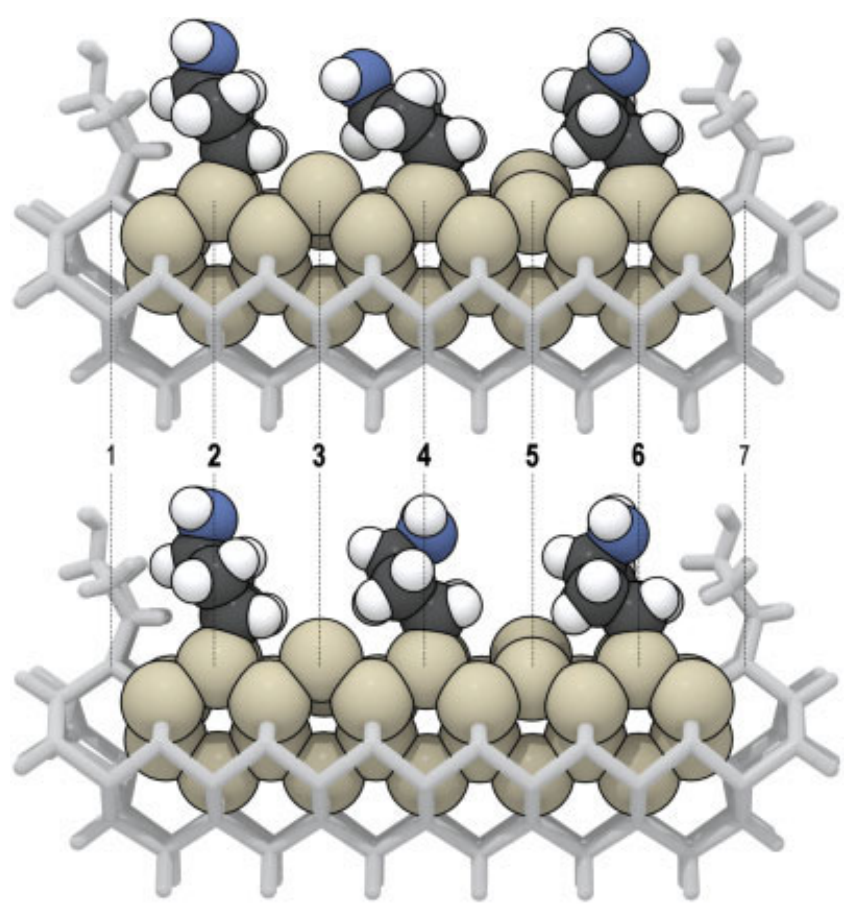

Figure 3. Two different conformations of $\mathrm{C}$-adsorbed $\mathrm{ACP}$ at the $\mathrm{Si}(100)$ surface. Both structures represent the aA_A_Aa case. The numbering of the dimer positions, as used in this work is also shown. Bold numbers are used for the testing positions. 
Table 1. ACP Adsorption Energies on the Clean Si(100) Surface or in Three-Spot Holes.

\begin{tabular}{|c|c|}
\hline Transition & $\Delta \mathrm{E}\left(\mathrm{kcal} \mathrm{mol}^{-1}\right)$ \\
\hline$a_{-} \ldots \ldots \_a \rightarrow a_{-} \ldots A_{-} a$ & -39.0 \\
\hline $\mathrm{a}_{-}{ }_{-}{ }_{-} \mathrm{a} \rightarrow \mathrm{a}_{-} \mathbf{A}_{-} \mathrm{a}_{\mathrm{a}}$ & -39.3 \\
\hline $\mathrm{a}_{-}{ }_{-}-\mathrm{a} \rightarrow \mathrm{a}_{-} \mathbf{A}_{-}{ }_{-} \mathrm{a}$ & -39.6 \\
\hline aAA___a $\rightarrow$ aAA_A_a & -38.9 \\
\hline $\mathrm{aA} \_-$Aa $\rightarrow$ aA_A_Aa & -38.5 \\
\hline a_ _ _AAa $\rightarrow$ a_A_AAa & -39.9 \\
\hline
\end{tabular}

Bold "A" represents the adsorbing molecule.

adsorbing at the fifth position, this effect is almost completely lost and we start getting the typical $-26 \mathrm{kcal} \mathrm{mol}^{-1}$ adsorption energy.

Since the ACP molecules adsorb in a bent conformation (see Fig. 3), we might have expected that adsorption would be different depending on whether the empty space is located on one side or the other of the adsorbing ACP. However, the adsorption energy for these two situations is similar, which shows that there is basically no difference concerning the relative position of the adsorbing molecule and the surface hole. Moreover, the existence of a two spot hole is energetically equivalent to a one spot hole, which is coherent with the results shown in Table 1. Comparing the $-26 \mathrm{kcal} \mathrm{mol}^{-1}$ adsorption energy with the one obtained for the clean surface, we realize that the proximity effect of two ACP molecules at the surface costs roughly $13 \mathrm{kcal} \mathrm{mol}^{-1}$.

Table 3 shows the energies corresponding to the adsorption of one ACP molecule in a one spot hole two positions away from another one spot hole. That is, between the adsorbing molecule and another spot on the surface there is one adsorbed ACP molecule. In this situation, the adsorption energy is only $-13 \mathrm{kcal} \mathrm{mol}^{-1}, 13 \mathrm{kcal} \mathrm{mol}^{-1}$ higher than the previous situation. This is what we would expect since we are now adsorbing the molecule in a one spot hole with one ACP molecule on each

Table 2. ACP Adsorption Energies for Situations Where the Adsorbing Molecule is Right Next to One Adsorbed ACP Molecule and to One Hole.

\begin{tabular}{ll}
\hline Transition & $\Delta \mathrm{E}\left(\mathrm{kcal} \mathrm{mol}^{-1}\right)$ \\
\hline aAA___a $\rightarrow$ aAA__Aa & -35.3 \\
aAAA__a $\rightarrow$ aAAA_Aa & -35.3 \\
aA___Aa $\rightarrow$ aA__AAa & -26.8 \\
aAA__Aa $\rightarrow$ aAA_AAa & -26.7 \\
a___AAa $\rightarrow$ a__AAAa & -24.7 \\
aA__AAa $\rightarrow$ aA_AAAa & -24.7 \\
aAAA__a $\rightarrow$ aAAAA_a & -25.3 \\
aAA_-_a $\rightarrow$ aAAA__a & -25.4 \\
aAA__Aa $\rightarrow$ aAAA_Aa & -25.4 \\
aA___Aa $\rightarrow$ aAA__Aa & -26.1 \\
aA__AAa $\rightarrow$ aAA_AAa & -25.9 \\
a___AAa $\rightarrow$ aA__AAa & -26.4 \\
a__AAAa $\rightarrow$ aA_AAAa & -26.3 \\
\hline
\end{tabular}

Bold "A" represents the adsorbing molecule.
Table 3. ACP Adsorption Energies for Situations Where Adsorption Occurs in One Hole Two Positions Away From Another Hole.

\begin{tabular}{lc}
\hline Transition & $\Delta \mathrm{E}\left(\mathrm{kcal} \mathrm{mol}^{-1}\right)$ \\
\hline aAA_A_a $\rightarrow$ aAA_AAa & -23.1 \\
aA_A_Aa $\rightarrow$ aA_AAAa & -13.1 \\
a_A_AAa $\rightarrow$ a_AAAAa & -12.1 \\
aAA_A_a $\rightarrow$ aAAAA_a & -11.8 \\
aA_A_Aa $\rightarrow$ aAAA_Aa & -13.0 \\
a_A_AAa $\rightarrow$ aAA_AAa & -13.2 \\
\hline
\end{tabular}

Bold "A" represents the adsorbing molecule.

side. Once more, adsorption at the sixth position is roughly $-10 \mathrm{kcal} \mathrm{mol}^{-1}$ lower than all the others, which shows that this effect is basically constant and predictable.

Table 4 shows the last five combinations studied in this work where we tested the adsorption of a molecule in a single spot hole on an otherwise completely occupied surface. This situation provides the last step needed to get a perfect $1 \mathrm{ML}$ surface coverage and we can see that this adsorption is barely exothermic, with an energy of only $-5 \mathrm{kcal} \mathrm{mol}^{-1}$. Once more this should be expected since there is very little space to fit one extra ACP molecule in one hole. The repulsions are high, which can be observed from the difference between the $-39 \mathrm{kcal} \mathrm{mol}^{-1}$ for the clean surface adsorption and this one. Even though the full surface coverage might be a thermodynamic possibility, kinetics seem to dictate otherwise. In previous studies, it was found that the C-adsorption of ACP involved the formation of a three-atom transition state ${ }^{23,35}$ that has been well characterized for cyclopentene, ${ }^{10}$ ethylene, ${ }^{36}$ and 1,3-dienes in general. ${ }^{37}$ This structure forces the ACP molecule to lay down relatively close to the surface occupying much more space than it does when completely adsorbed. By taking this mandatory transition step into consideration, we clearly see that adsorption in a single spot hole should be indeed impossible and that even the previous combinations shown in Tables 2 and 3 may be affected by this.

It is interesting to notice that the energy for the first result in Table 4 is quite similar to the results in Table 3 and this is indeed understandable because the two situations are similar. A single ACP molecule is located between the adsorbing molecule and a hole on the surface. In this case, it is not exactly a hole but the end of the cluster, although the effect seems to be basically the same. This helps to predict what can happen with new combinations. For instance, the first two results in Table 2 point

Table 4. ACP Adsorption Energies When the Adsorbing Molecule Adsorbs in a Single Spot Hole in the Surface.

\begin{tabular}{lc}
\hline Transition & $\Delta \mathrm{E}\left(\mathrm{kcal} \mathrm{mol}^{-1}\right)$ \\
\hline aAAAA_a $\rightarrow$ aAAAAAa & -15.3 \\
aAAA_Aa $\rightarrow$ aAAAAAa & -5.3 \\
aAA_AAa $\rightarrow$ aAAAAAa & -4.0 \\
aA_AAAa $\rightarrow$ aAAAAAa & -5.2 \\
a_AAAAa $\rightarrow$ aAAAAAa & -5.2 \\
\hline
\end{tabular}

Bold "A" represents the adsorbing molecule. 
to the fact that the adsorption energy for a combination such as _AA_ should be roughly $-35 \mathrm{kcal} \mathrm{mol}^{-1}$; the first result in Table 3 indicates that the _AAA_ situation should have an adsorption energy of roughly $-23 \mathrm{kcal} \mathrm{mol}^{-1}$.

With all the combinations studied here, we now have a proper description of most possible scenarios for the $\mathrm{C}$-adsorbed trans-ACP molecule on the $\mathrm{Si}(100)$ surface. The most important conclusion is that it is impossible to obtain a $1 \mathrm{ML}$ ACP covered $\mathrm{Si}(100)$ surface. Indeed, STM images already showed that the typical cyclopentene functionalized $\mathrm{Si}(100)$ surface is mostly filled with molecules at a distance that is around double the distance seen between the silicon dimers. ${ }^{32,33}$ We clearly see that forming the $0.5 \mathrm{ML}$ surface seems straightforward and that each molecule adsorbs as if the surface was completely clean. However, over $0.5 \mathrm{ML}$, the situation gets more complicated due to the molecules proximity and the adsorption energies become less favorable. Moreover, steric hindrance avoiding the formation of the typical intermediate state seems to avoid any further adsorption over $0.5 \mathrm{ML}$.

These results may seem to be in contradiction with previous theoretical works using periodic cell calculations that present adsorption energies per molecule for the 1ML coverage of around $-25 \mathrm{kcal} \mathrm{mol}^{-1}$ for $\mathrm{ACP}^{35}$ and $-27 \mathrm{kcal} \mathrm{mol}^{-1}$ for cyclopentene. ${ }^{10}$ However, if we use our results to calculate the adsorption energy per ACP molecule for the full coverage surface, we obtain a value of $-26.5 \mathrm{kcal} \mathrm{mol}^{-1}$ per ACP molecule adsorbed that is in perfect agreement with those previous works. Therefore, care must be taken when analyzing all-system average adsorption energies.

One final characteristic of the system that we can get from the obtained energies, mainly the ones shown in Tables 2 and 3, is the flexibility that the adsorbed ACP molecules seem to have. For instance, in cases such as _AAA_ with a $-23 \mathrm{kcal} \mathrm{mol}^{-1}$ adsorption energy, as opposed to the aAAAAAa with only $-4 \mathrm{kcal} \mathrm{mol}^{-1}$, the energy difference is probably associated with the mobility of the ACP molecules that adjust to the adsorbing molecule giving it more space and reducing the repulsive interactions between them. And even though this structure might not form experimentally, it shows that there is significant mobility of the ACP molecules. This may be very relevant for surface protection situations because, while additional ACP molecules may not get in, several smaller molecules such as water and oxygen can probably reach the surface. In other words, the ACP functionalization of the silicon surface may not be good enough for surface protection against smaller molecules.

It is relevant to note that these results correspond to potential energy calculations for adsorption processes, therefore not containing entropic effects. In any case, even though free energy calculations may differ from these values, we believe that the general conclusions are still applicable. In a related work, ${ }^{35}$ the comparison between $\Delta E$ and $\Delta G$ shows similar trends.

\section{Conclusions}

We have shown how the ONIOM methodology (QM/QM) calculation method can be used to successfully study surface coverage. Because of its size, the ACP molecule can be easily adsorbed without a significant perturbation until a $0.5 \mathrm{ML}$ surface coverage is achieved. However, there are significant repulsive interactions when the molecules come too close together on higher surface densities. In fact, when adsorbing one ACP molecule in a single-spot hole on an otherwise completely covered surface, we get adsorption energies approximate to zero. Moreover, due to the difficulty to achieve the typical transition state involved in the $[2+2]$ cycloaddition reaction, coverages over $0.5 \mathrm{ML}$ should be very difficult to get.

Finally, we have shown the suitability of hybrid QM/QM calculations used on large clusters. The energies seem reliable and most of the time concur with experimental works and much heavier calculation studies.

\section{Acknowledgment}

H. R. R. S. gratefully acknowledges access to the Centres de Computació i Comunicacions de Catalunya (CEPBA-CESCA).

\section{References}

1. Onclin, S.; Ravoo, B. J.; Reinhoudt D. N. Angew Chem 2005, 44, 6282.

2. Chaki, N. K.; Vijayamohanan, K. Biosens Bioelectron 2002, 17, 1.

3. Senaratne, W.; Andruzzi, L.; Ober, C. K. Biomacromolecules 2005, 6, 2427.

4. Frenkel, D.; Smit, B. Understanding Molecular Mechanics-From Algorithms to Applications; Academic Press: San Diego, 2002.

5. Gunsteren, W. F. V.; Berendsen, H. J. C. Angew Chem Int Ed 1990, 29, 992.

6. Thiel, W. In Modern Methods and Algorithms of Quantum Chemistry; Grotendorst, J., Ed.; John von Neumann Institute for Computing, Jülich, 2002.

7. Thiel, W. Adv Chem Phys 1997, 93, 703.

8. Dixon, S. L.; Merz, K. M., Jr. J Chem Phys 1997, 107, 879.

9. Deák, P.; Snyder, L. C.; Singh, R. K.; Corbett, L. W. Phys Rev B 1987, 36, 9612.

10. Cho, J.-H.; Kleinman, L. Phys Rev B 2001, 64, 235420.

11. Hohenberg, P.; Kohn, W. Phys Rev B 1964, 136, B864.

12. Kohn, W.; Sham, L. J. Phys Rev A 1965, 140, A1133.

13. Parr, R. G.; Yang, W. Density-Functional Theory of Atoms and Molecules; Oxford University Press: Oxford, 1989.

14. Cao, X.; Hamers, R. J. J Vac Sci Technol Part B Microelectron Nanometer Struct 2002, 20, 1614.

15. Lu, W.; Schmidt, W. G.; Bernholc, J. Phys Rev B 2003, 68, 115327.

16. Head-Gordon, M.; Pople, J. A.; Frisch, M. J. Chem Phys Lett 1988, $153,503$.

17. Pople, J. A.; Krishnan, R.; Schlegel, H. B.; Binkley, J. S. Int J Quantum Chem 1978, 14, 545.

18. Kudin, K. N.; Scuseria, G. E. Phys Rev B 2000, 61, 16440.

19. Maseras, F.; Morokuma, K. J Comput Chem 1995, 16, 1170.

20. Morokuma, K.; Vreven, T. J Comput Chem 2000, 21, 1419.

21. Dapprich, S.; Komáromi, I.; Byun, K. S.; Morokuma, K.; Frisch, M. J. J Mol Struct 1999, 461, 1.

22. Santos, H. R. R.; Ramos, M. J.; Gomes, J. A. N. F. C R Chim 2005, $8,1461$.

23. Santos, H. R. R.; Ramos, M. J.; Gomes, J. A. N. F. Phys Rev B 2005, 72, 075445 .

24. Becke, A. D. J Chem Phys 1993, 98, 5648.

25. Dunning, T. H., Jr.; Hay, P. J. Modern Theoretical Chemistry; Plenum: New York, 1977. 
26. Rappé, A. K.; Smedley, T. A.; Goddard, W. A., III. J Phys Chem 1981, 85, 1662.

27. Dewar, M. J. S.; Zoebisch, E. G.; Healy, E. F.; Stewart, J. J. P. J Am Chem Soc 1985, 107, 3902.

28. Lin, Z.; Strother, T.; Cai, W.; Cao, X.; Smith, L. M.; Hamers, R. J. Langmuir 2002, 18, 788.

29. Engel, T. Surf Sci Rep 1993, 18, 93.

30. Thiel, P. A.; Madey, T. E. Surf Sci Rep 1987, 7, 211.

31. Dabrowski, J.; Müssig, H.-J. Silicon Surfaces and Formation of Interfaces; World Scientific: Singapore, 2000.

32. Hovis, J. S.; Liu, H.; Hamers, R. J Appl Phys A 1998, 66, S553.

33. Hamers, R. J.; Hovis, J. S.; Greenlief, C. M.; Padowitz, D. F. Jpn J Appl Phys 1999, 38(Part 1, No. 6B), 3879.

34. Frisch, M. J.; Trucks, G. W.; Schlegel, H. B.; Scuseria, G. E.; Robb, M. A.; Cheeseman, J. R.; Montgomery, J. A., Jr.; Vreven, T.; Kudin, K. N.; Burant, J. C.; Millam, J. M.; Iyengar, S. S.; Tomasi, J.; Barone, V.; Mennucci, B.; Cossi, M.; Scalmani, G.; Rega, N.; Petersson, G. A.; Nakatsuji, H.; Hada, M.; Ehara, M.; Toyota, K.; Fukuda, R.; Hase- gawa, J.; Ishida, M.; Nakajima, T.; Honda, Y.; Kitao, O.; Nakai, H.; Klene, M.; Li, X.; Knox, J. E.; Hratchian, H. P.; Cross, J. B.; Bakken, V.; Adamo, C.; Jaramillo, J.; Gomperts, R.; Stratmann, R. E.; Yazyev, O.; Austin, A. J.; Cammi, R.; Pomelli, C.; Ochterski, J. W.; Ayala, P. Y.; Morokuma, K.; Voth, G. A.; Salvador, P.; Dannenberg, J. J.; Zakrzewski, V. G.; Dapprich, S.; Daniels, A. D.; Strain, M. C.; Farkas, O.; Malick, D. K.; Rabuck, A. D.; Raghavachari, K.; Foresman, J. B.; Ortiz, J. V.; Cui, Q.; Baboul, A. G.; Clifford, S.; Cioslowski, J.; Stefanov, B. B.; Liu, G.; Liashenko, A.; Piskorz, P.; Komaromi, I.; Martin, R. L.; Fox, D. J.; Keith, T.; Al-Laham, M. A.; Peng, C. Y.; Nanayakkara, A.; Challacombe, M.; Gill, P. M. W.; Johnson, B.; Chen, W.; Wong, M. W.; Gonzalez, C.; Pople, J. A. Gaussian 94, Revision E.2, Gaussian: Wallingford, CT, 2003.

35. Festa, G.; Cossi, M.; Barone, V.; Cantele, G.; Ninno, D.; Iadonisi, G. J Chem Phys 2005, 122, 184714.

36. Liu, H.; Hamers, R. J. J Am Chem Soc 1997, 119, 7593.

37. Hovis, J. S.; Liu, H.; Hamers, R. J. J Phys Chem B 1998, 102, 6873. 